\section{A NEW MICROSTRIP PATCH ANTENNA FOR MOBILE COMMUNICATIONS AND BLUETOOTH APPLICATIONS}

Binu Paul, S. Mridula, C. K. Aanandan, and P. Mohanan Center for Research in Electro-Magnetics and Antennas Department of Electronics

Cochin University of Science and Technology

Cochin 22, Kerala, India

\section{Received 8 November $200 /$}

ABSTRACT: A compact multiband planar octagonal-shaped microstrip antenna simultaneously suitable for mobile communication and blue tooth application is presented. The antenna provides sufficient isolation between the two operating bands and an area reduction of $\approx 29 \%$ with respect to a circular patch operating in the same band. (c) 2002 Wiley Periodicals, Inc. Microwave Opt Technol Lett 33: 285-286, 2002; Published online in Wiley InterScience (www.interscience.wiley.com). DOI 10.1002/mop. 10297

Key words: microstrip antennas; dual polarization; mobile communication; blue tooth; multiband

\section{INTRODUCTION}

Current mobile wireless terminals employ either separate antennas or a combination of the two in one embodiment, in order to achieve multiband coverage of cellular and PCS bands. A single low-cost mobile terminal equipped to operate in several regions of the world, each with different operating frequency, is essential for future generation mobile wireless systems. Therefore, there is a clear and immediate need for a low-cost, compact, highly reliable, integrated antenna with no moving parts, capable of operating over multiple bands. The demand for higher capacity on wireless communication systems led to the development of a dual polarized antenna. The parameters of interest are low cross polarization, high impedance bandwidth, and small physical size. A cross polarization better than $-25 \mathrm{~dB}$ ensures sufficient suppression of cross talk in polarization diversity systems. A large impedance bandwidth is preferred in portable devices to compensate for coupling effects of

\section{Top View}

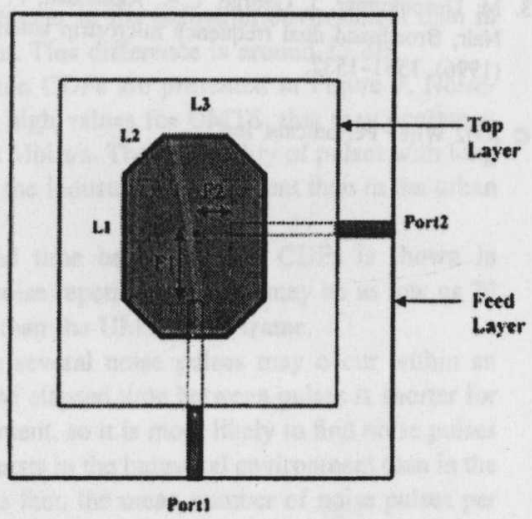

Side View

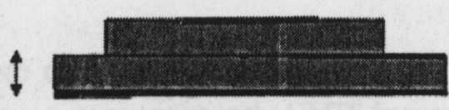

Figure 1 The proposed antenna geometry

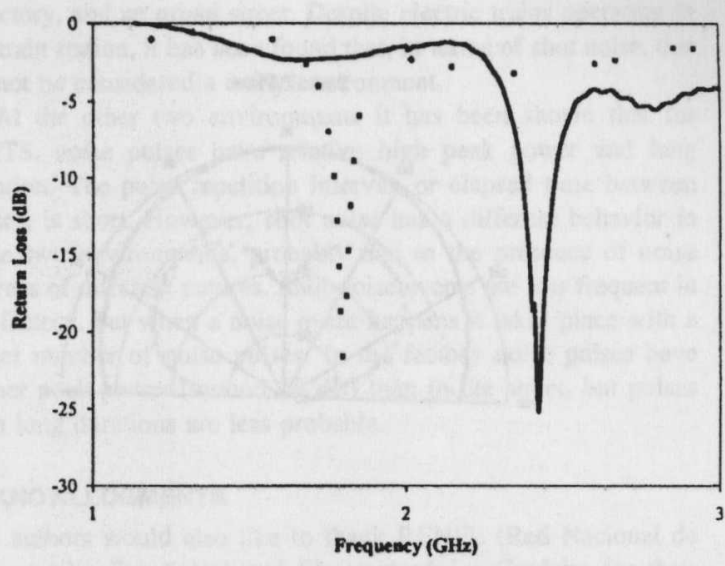

Figure 2 Variation of return loss with frequency. Dotted line, Port 1; solid line, Port 2

nearby objects and scatterers. A small physical size is needed because the devices themselves are small.

A compact pentagon microstrip antenna for a single operating frequency has been proposed for circular polarization [1]. Dual band frequency characteristics using microstrip patches require two elements of different dimensions resonating in the required frequency bands. The potential of an irregular-shaped planar antenna has been proposed for multiband operation [2]. Multiband operation is obtained by perturbing the shape of a conventional microstrip patch antenna in a prescribed manner [3]. In this Letter a compact octagonal microstrip antenna providing all the above desirable radiation characteristics is presented.

\section{ANTENNA STRUCTURE}

The geometry of the octagon-shaped patch is shown in Figure 1. The patch dimensions are $L_{1}=24 \mathrm{~mm}, L_{2}=15.5 \mathrm{~mm}, L_{3}=10$ $\mathrm{mm}$. The antenna is fabricated on a substrate of dielectric constant $\varepsilon_{r}=4.28$ and thickness $h=0.16 \mathrm{~cm}$. The two ports are energized electromagnetically with the use of two $50 \Omega$ perpendicular microstrip lines. The feed lines are also fabricated on a similar substrate. The feed locations are optimized with IE3D software and finally trimmed experimentally as $F_{1}=12 \mathrm{~mm}$ and $F_{2}=$ $3.5 \mathrm{~mm}$ from the geometric center of the patch. The frequencies

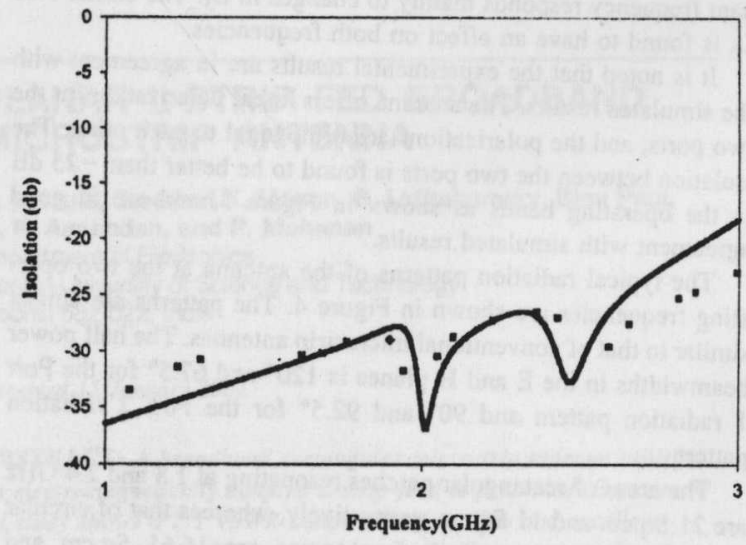

Figure 3 Isolation in the operating band. Dotted line, experimenta results; solid line, simulated results 

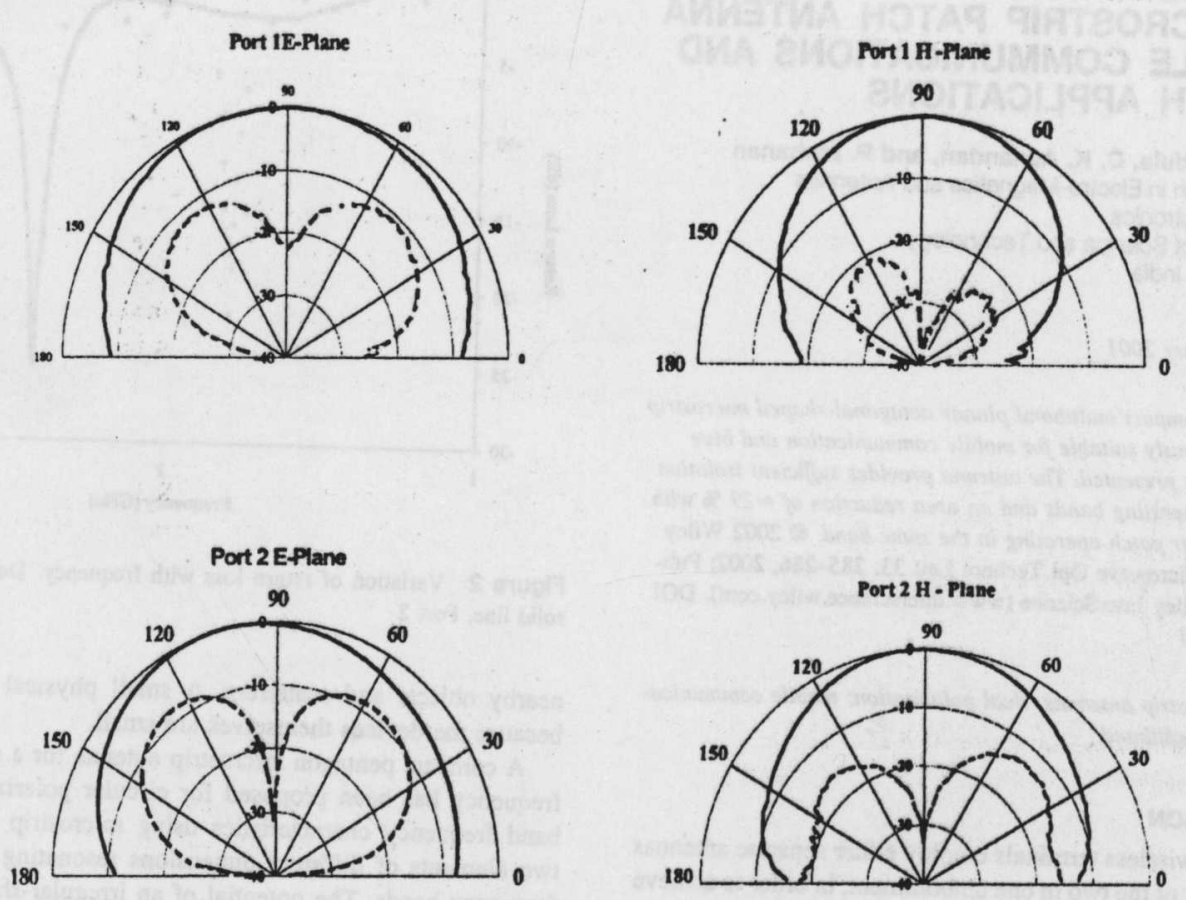

Figure 4 Radiation patterns of the proposed antenna. Solid line, co-polarization; dashed line, cross-polarization

chosen for the study are 1.8 and $2.4 \mathrm{GHz}$, respectively, for Ports 1 and 2 to accommodate both the mobile communication and the blue tooth frequencies, respectively.

\section{EXPERIMENTAL RESULTS}

The variation of return loss with frequency of the octagonal patch antenna is shown in Figure 2. The operating band of Port 1 is from 1.7625 to $1.8313 \mathrm{GHz}$ and that of Port 2 is from 2.3625 to 2.475 GHz. The 2:1 VSWR impedance bandwidths obtained are 3.82 and $4.65 \%$ for Ports 1 and 2 , operating in the first and the second bands, respectively. It is observed experimentally that the dimensions $L_{1}$ and $L_{2}$ have a profound influence on the resonant frequencies of Ports 1 and 2, respectively. The Port 1 resonant frequency responds mainly to changes in $L_{1}$ and the Port 2 resonant frequency responds mainly to changes in $L_{3}$. The corner side $L_{2}$ is found to have an effect on both frequencies.

It is noted that the experimental results are in agreement with the simulated results. The antenna offers linear polarization for the two ports, and the polarizations are orthogonal to each other. The isolation between the two ports is found to be better than $-25 \mathrm{~dB}$ in the operating bands as shown in Figure 3, and are in good agreement with simulated results.

The typical radiation patterns of the antenna at the two operating frequencies are shown in Figure 4. The patterns are almost similar to that of conventional microstrip antennas. The half power beamwidths in the $\mathrm{E}$ and $\mathrm{H}$ planes is $120^{\circ}$ and $67.5^{\circ}$ for the Port 1 radiation pattern and $90^{\circ}$ and $92.5^{\circ}$ for the Port 2 radiation pattern.

The areas of rectangular patches resonating at 1.8 and $2.4 \mathrm{GHz}$ are $21 \mathrm{Sq} . \mathrm{cm}$ and 11 Sq.cm, respectively, whereas that of circular patches resonating at these frequencies are $16.61 \mathrm{Sq} . \mathrm{cm}$ and $9.2354 \mathrm{Sq} . \mathrm{cm}$, respectively. The proposed octagonal patch antenna occupics an area of only $11.8 \mathrm{Sq} . \mathrm{cm}$, resulting in an area reduction of $50.83 \%$ with respect to the rectangular patch area and $28.96 \%$ with respect to the circular patch area.

\section{CONCLUSIONS}

The proposed octagon-shaped patch antenna has demonstrated its potential for multiband operation and hence its suitability for installation in mobile wireless terminals as in GPS and WLAN. It will have a significant impact on reducing the size, cost, and reliability of the mobile terminals.

\section{REFERENCES}

1. H.D. Chen and W.S. Chen, Compact pentagon microstrip antenna with circular polarization. Microwave Opt Technol Lett 6 (2001), 370-372.

2. S. Maci and G. B. Gentili, Dual frequency patch antennas. IEEE Antennas Propagat Mag 39 (1997), 13-20.

3. M. Deepukumar, J. George, C.K. Aanandan, P. Mohanan, and K.G. Nair, Broadband dual frequency microstrip antenna. Electron Lett 32 (1996), 1531-1532.

(c) 2002 Wiley Periodicals, Inc. 


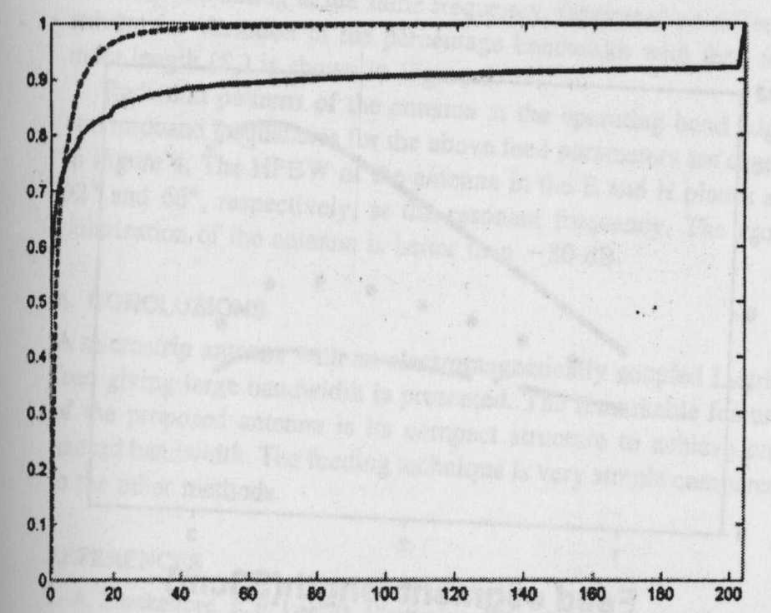

$\mu$

Figure 8 Elapsed time between pulses cumulative distribution. Urban environment (solid line) and industrial environment (dashed line)

Due to the burst nature of the shot noise there are, on average, more than one noise pulse per noise register. The presence of strong impulsive noise sources such as motorcycles in the environment gives rise to noise registers with many noise pulses, as can be seen in Figure 5. The elapsed time between pulses can only be measured if there are two or more noise pulses per register. If there is only one noise pulse in the register, the elapsed time cannot be calculated, but it can be concluded that it is larger than $204 \mu \mathrm{s}$.

The measurements show that, as expected, the in-phase and quadrature components are uncorrelated but dependent, and that the noise phase is uniformly distributed in the $[0,2 \pi]$ interval.

The peak amplitude cumulative distribution functions (CDFs) of the power at the antenna output is shown in Figure 6. Some noise pulses occur with very high peik power, compared to the reference sensitivity at a UMTS base station receiver $[8](-121$ $\mathrm{dBm}$ ) or even additive white Gaussian noise (AWGN) levels given in the dynamic range specification [8]. Impulsive noise power is very high, even for a CDMA system to operate correctly. The noise peak power is higher in the industrial environment than in the urban environment. This difference is around $15 \mathrm{~dB}$.

Noise pulse duration CDFs are presented in Figure 7. Noisepulse durations reach high values for UMTS, that may operate at data rates as high as $2 \mathrm{Mbits} / \mathrm{s}$. The probability of pulses with long duration is smaller in the industrial environment than in the urban environment.

Finally the elapsed time between pulse CDFs is shown in Figure 8. Impulsive noise repetition interval may be as low as 20 $\mu \mathrm{s}$, which is smaller than the UMTS data frame.

As a consequence several noise pulses may occur within an UMTS data frame. The elapsed time between pulses is shorter for the industrial environment, so it is more likely to find noise pulses to occur grouped in bursts in the industrial environment than in the urban environment. In fact, the mean number of noise pulses per register is 5.9 in the urban environment and 23.4 in the industrial environment.

\section{SUMMARY AND CONCLUSIONS}

A shot-noise measurement campaign in the UMTS frequency band has been conducted in three different environments: a train station, 
a factory, and an urban street. Despite electric trains operating in the train station, it has been found that, in terms of shot noise, this cannot be considered a noisy environment.

At the other two environments it has been shown that for UMTS, noise pulses have relative high peak power and long duration. The pulse repetition interval, or elapsed time between pulses, is short. However, shot noise has a different behavior in these two environments, probably due to the presence of noise sources of different natures. Shot-noise events are less frequent in the factory, but when a noise event happens it takes place with a larger number of noise pulses. In the factory noise pulses have higher peak power (around $15 \mathrm{~dB}$ ) than in the street, but pulses with long durations are less probable.

\section{ACIKNOWLEDGMENTS}

The authors would also like to thank RENFE (Red Nacional de Ferrocarriles Españoles) and Electromecánica Cerdeira for their help in the measurements at the train station and at the factory, respectively.

\section{REFERENCES}

1. E.N. Gilbert and H.O. Pollak, Amplitude distribution of shot noise, Bell Syst Tech J (1960), 333-350.

2. \$. Miyamoto, M. Katayama, and N. Morinaga, Performance analysis of QAM systems under class $A$ impulsive noise environment, IEEE Trans Electromagn Compat EC-37 (1995), 260-267.

3. 3. Miyamoto, M. Katayama, and N. Morinaga, Receiver design using the dependence between quadrature components of impulsive radio noise, in Proceedings of the IEEE International Conference on Communications, Seattle, June, 1995.

4. K.L. Blackard and T.S. Rappaport, Measurements and models of radio Irequency impulsive noise for indoor wireless communications, IEEE J Selected Areas Commun SAC-11 (1993), 991-1001.

5. D. Parsons, The mobile radio propagation channel, Pentech Press, London, 1992, Chap. 9.

6. M.G. Sánchez, L. de Haro, M. Calvo, A. Mansilla, C. Montero, and D. Oliver, Impulsive noise measurements and characterization in a UHF digital TV channel, IEEE Trans Electromagn Compat EC-41 (1999), 124-136.

7. W.R. Lauber and J.M. Bertrand, Statistics of motor ignition noise at VHF/UHF, IEEE Trans Electromagn Compat EC-4 (1999), 257-259.

8. 3GPP TS 25.104 V5.0.0 (2001-09) Technical Specification. 3rd Generation Partnership Project; Technical Specification Group Radio Access Networks; UTRA (BS) FDD; Radio transmission and Reception (Release 5). 